VOL. LX/LXI DÉDIÉ À M. ANTONI ZYGMUND 1990

\title{
REGULARITY OF THE POISSON KERNEL AND FREE BOUNDARY PROBLEMS
}

\author{
BY \\ DAVID JERISON* (CAMBRIDGE, MASSACHUSETTS)
}

Introduction. In this note we will consider the relationship between the regularity of the boundary of a domain and the regularity of its Poisson kernel. Because the Poisson kernel is the normal derivative of Green's function, one can expect the Poisson kernel to be differentiable of order one less than the differentiability of the boundary. For instance, it is well known that if the boundary is $C^{k+1, \alpha}$, for some integer $k \geq 0$ and $0<\alpha<1$, then the Poisson kernel is $C^{k, \alpha}$ (as a function of the variable in the boundary). We will focus on the converse, which says roughly that if the Poisson kernel is $C^{k, \alpha}$, then the boundary is $C^{k+1, \alpha}$. We will also consider the case in which the Poisson kernel is merely continuous.

The converse problem is a free boundary problem because we are asking for the regularity of the boundary given the regularity of the 0th and first derivative of a harmonic function (Green's function). In order to prove the converse we will make use of fundamental free boundary estimates due to Caffarelli [3]. (See also [1].) The case of higher regularity $(k \geq 2)$ was proved by Kinderlehrer and Nirenberg [11]. But in the case where data yield boundary regularity of order less than two, such as when the Poisson kernel is merely $C^{\alpha}$, for some $\alpha<1$, no proof of the sharp order of regularity appears in the literature $\left({ }^{1}\right)$. This is because it is necessary to use fractional derivatives and second differences, techniques associated to Professor Zygmund and the Zygmund class $\Lambda_{*}$ (see [20, p. 263, Theorem 5.1]).

Thus we will supply some Schauder-type boundary estimates which, together with Caffarelli's deep result, complete the picture of the simple re-

* Supported by NSF grant DMS-8804582 and a Presidential Young Investigator Award (DMS-8451770). This research was begun at the Mathematical Sciences Research Institute in Berkeley.

$\left({ }^{1}\right)$ However, the techniques of Lieberman $[14,15]$ also yield a proof. Moreover, Kinderlehrer, Nirenberg, and Spruck [12] have proved real analyticity for a related nonlinear problem assuming solutions are a priori $C^{1}$. Finally, related homogeneous linear problems are treated by Pipher [17]. 
lationship between $C^{\alpha}$ regularity of the Poisson kernel and boundary regularity. The only novelty in the $C^{\alpha}$ case is that we make inhomogeneous Schauder-type estimates in function spaces involving some blow-up at the boundary. With the help of a Rellich formula, we will also prove the sharp endpoint estimate when the Poisson kernel is $C^{0}$. Finally, we will discuss open questions that are part of the program of understanding free boundaries as clearly as we understand minimal surfaces.

2. Notations and main results. Consider a domain above the graph of a Lipschitz function. Let $\varphi: \mathbf{R}^{n} \rightarrow \mathbf{R}$ satisfy the Lipschitz condition $\left|\varphi(x)-\varphi\left(x^{\prime}\right)\right| \leq A\left|x-x^{\prime}\right|$ for all $x, x^{\prime} \in \mathbf{R}^{n}$. Set $\Omega=\left\{\left(x_{0}, x\right) \in \mathbf{R} \times \mathbf{R}^{n}\right.$ : $\left.x_{0}>\varphi(x)\right\}$. For each $\alpha>0$, define

$$
\Lambda(\alpha)=\left\{f \in C\left(\mathbf{R}^{n}\right):\|f\|_{\alpha}=\sup _{\substack{x \in \mathbf{R}^{n} \\ r>0}} r^{-\alpha} \inf _{\boldsymbol{P}} \sup _{B(x, r)}|f(y)-P(y)|<\infty\right\}
$$

where the infimum is over all polynomials of degree $\leq \alpha$. This is the same (modulo polynomials) as the class $\Lambda_{\alpha}$ of [19]. In particular, $\Lambda(1)$ is the Zygmund class. There is a harmonic function $u$ (unique up to a constant multiple) such that $u>0$ in $\Omega, u \in C(\bar{\Omega})$. and $u=0$ on $\partial \Omega$ (see $[8,9]$ ). This function should be thought of as Green's function with pole at $\infty$. It follows from Dahlberg's theorem [4] and related results that $\nabla u$ has nontangential limits a.e. on $\partial \Omega$. Define $h(Q)=-N_{Q} \cdot \nabla u(Q)$, where $N_{Q}$ is the outer unit normal. (Thus $h(Q)$ is defined almost everywhere and is nonnegative.) We will refer to $h(Q)$ as the Poisson kernel. Indeed, the ordinary Poisson kernel with finite pole is a multiple of this function by a nonzero factor that is smoother than $h$. Moreover, the Poisson kernel on a bounded domain whose boundary overlaps with $\partial \Omega$ on some fixed ball $B_{r}$ will agree up to a similar smoother nonzero factor at least on $B_{r / 2} \cap \partial \Omega$. Thus the regularity of $h$ is the same as the regularity of the ordinary Poisson kernel.

THEOREM 1. Suppose that $\varphi$ is a Lipschitz function. Then for all $\alpha>0$, $\varphi \in \Lambda(\alpha+1)$ if and only if $\log h(\varphi(x), x) \in \Lambda(\alpha)$.

Notice that locally the statement $\log h \in \Lambda(\alpha)$ is equivalent to $h \in$ $\Lambda(\alpha)$ and $h>0$. Also note that one could also express this statement without using graph coordinates by saying that $\log h(Q)$ agrees with the restriction to $\partial \Omega$ of a $\Lambda(\alpha)$ function in $\mathbf{R}^{n+1}$. Furthermore, because we are assuming that $\varphi$ is Lipschitz, Theorem 1 only has interest for distance less than 1 . Indeed, $\log h(\varphi(x), x)$ grows at most like $C \log |x|$ as $x \rightarrow \infty$, and the $\Lambda(\alpha)$ and $\Lambda(\alpha+1)$ estimates for $h$ and $\varphi$ on large balls (radius $\geq 1)$ are automatically satisfied. The forward implication: $\varphi \in \Lambda(\alpha+$ 1) implies $h \in \Lambda(\alpha)$ is well known. It follows from the standard layer potential technique [6]. The proof of the reverse implication will involve 
second differences even when $\alpha<1$ because $1+\alpha>1$.

Let $f_{B}$ denote the average value of $f$ on $B$. The space of vanishing mean oscillation is

$$
\mathrm{VMO}=\left\{f \in L^{2}\left(\mathbf{R}^{n}\right): \lim _{r \rightarrow 0} \sup _{\substack{x \in R^{n} \\ s \leq r}} \frac{1}{s^{n}} \int_{B(x, s)}\left|f(y)-f_{B(x, s)}\right| d y=0\right\} .
$$

VMO is the closure in BMO of the subspace of uniformly continuous functions in BMO.

THEOREM 2. Suppose that $\varphi$ is a Lipschitz function. If, in addition, $\nabla \varphi$ is uniformly continuous, then $\log h(\varphi(x), x) \in$ VMO. Conversely, if $h(\varphi(x), x)$ is uniformly continuous, then $\nabla \varphi \in$ VMO.

The first statement is due to Jerison and Kenig [10].

Let us recall Caffarelli's result [3, part I].

TheOREM 3. Let $\varphi$ be a Lipschitz function. Suppose that $\alpha>0$ and that $\log h(\varphi(x), x) \in \Lambda(\alpha)$. Then there exists $\varepsilon>0$ such that $\nabla \varphi \in \Lambda(\varepsilon)$.

Our goal is to improve the conclusion to $\nabla \varphi \in \Lambda(\alpha)$.

In the limiting case Caffarelli's method also yields a result. Recall that the little-o Zygmund class is

$$
\lambda_{*}=\left\{f \in \Lambda(1): \lim _{r \rightarrow 0} \sup _{x} \inf _{P} r^{-1} \sup _{B(x, r)}|f(y)-P(y)|=0\right\}
$$

where the infimum is taken over polynomials of degree $\leq 1$.

THEOREM 4 (proved by the method of [3]). Let $\varphi$ be a Lipschitz function. If $\log h(\varphi(x), x)$ is uniformly continuous, then $\varphi \in \lambda_{*}$.

(It is well known that $\nabla \varphi \in$ VMO implies $\varphi \in \lambda_{*}$.)

3. Linear estimates. Suppose that $0<s<1$. Let $a_{i j}(Y), Y \in$ $\mathbf{R}_{+} \times \mathbf{R}^{n}$, be coefficients in $\Lambda^{+}(s)$, the restriction to $\mathbf{R}_{+} \times \mathbf{R}^{n}$ of functions in $\Lambda(s)$ of $\mathbf{R}^{n+1}$. Suppose that for some $\lambda>0, \sum_{i, j=0}^{n} a_{i j}(Y) \xi_{i} \xi_{j} \geq \lambda|\xi|^{2}$ for all $\xi \in \mathbf{R}^{n+1}$. Suppose that $b_{0}, b_{1}, \ldots, b_{n} \in \Lambda(s)$ and $b_{0}(Y) \geq c_{0}>0$. Let $I$ denote the unit cube $-1 \leq y_{j} \leq 1, j=0,1, \ldots, n$.

Let $-1<\alpha-\delta<0, \delta>0$. Define

$$
\begin{aligned}
B_{\delta}^{+}(\alpha)=\left\{f \in C\left(\mathbf{R}_{+} \times \mathbf{R}^{n}\right): \mid f\left(x_{0}, x\right)\right. & -f\left(x_{0}^{\prime}, x^{\prime}\right) \mid \\
& \left.\leq C\left(x_{0}^{\alpha-\delta}+\left|x_{0}^{\prime}\right|^{\alpha-\delta}\right)\left|x-x^{\prime}\right|^{\delta}\right\} .
\end{aligned}
$$

For $E \subset \mathbf{R}^{n+1}, B_{\delta}^{+}(\alpha, E)$ denotes the restriction to $E \cap\left(\mathbf{R}_{+} \times R^{n}\right)$ of $B_{\delta}^{+}(\alpha)$. Similarly $\Lambda(\alpha, E)$ denotes the restriction to $E \cap\left(\{0\} \times R^{n}\right)$ of $\Lambda(\alpha)$.

The main result of this section is 
Theorem A. Suppose $\sum_{i, j=0}^{n} a_{i j}(Y) w_{i j}(Y)=R(Y), Y \in \mathbf{R}_{+} \times \mathbf{R}^{n}$, and $\sum_{j=0}^{n} b_{j}(0, y) w_{j}(0, y)=f(y), y \in R^{n}$, where $w_{i}$ and $w_{i j}$ are the corresponding derivatives of $w$. Then

$$
\begin{aligned}
\left\|\nabla^{2} w\right\|_{B_{s}^{+}(s-1, I)} \leq & C\left(\|R\|_{B_{\delta}^{+}(s-1,2 I)}+\|\nabla w\|_{B_{b}^{+}(s-1,2 I)}\right. \\
& \left.+\|w\|_{B_{\delta}^{+}(s-1,2 I)}+\|w\|_{\Lambda(s, 2 I)}+\|f\|_{\Lambda(s, 2 I)}\right) .
\end{aligned}
$$

Furthermore, the constant $C$ depends only on $\lambda, c_{0},\left\|a_{i j}\right\|_{\Lambda^{+}(s)}$ and $\left\|b_{j}\right\|_{\Lambda(s)}$, $i, j=0,1, \ldots, n$.

Before we prove Theorem A we will explain the significance of $B_{\delta}^{+}(\alpha)$ by

Proposition 1. If $-1<\alpha-\delta<0$, then $\|F(0, \cdot)\|_{\Lambda(\alpha+1)} \leq C\|\nabla F\|_{B_{b}^{+}(\alpha)}$.

Proof. Let $x, t \in \mathbf{R}^{n}$. Then $\nabla F \in B_{\delta}^{+}(\alpha)$ implies $\mid \nabla F(r, x+t)-$ $\left.\nabla F(r, x)\left|\leq C r^{\alpha-\delta}\right| t\right|^{\delta}$. Therefore,

$$
\begin{aligned}
& \mid F(|t|, x+t)+F(|t|, x-t)-2 F(|t|, x)-(F(0, x+t)+F(0, x-t)-2 F(0, x)) \mid \\
&=\left|\int_{0}^{|t|}\left[\left(\partial_{0} F(r, x+t)-\partial_{0} F(r, x)\right)+\left(\partial_{0} F(r, x-t)-\partial_{0} F(r, x)\right)\right] d r\right| \\
& \leq C \int_{0}^{|t|} r^{\alpha-\delta}|t|^{\delta} d r=C|t|^{1+\alpha} .
\end{aligned}
$$

Next,

$$
\begin{aligned}
\mid F(|t|, x+t)+ & F(|t|, x-t)-2 F(|t|, x) \mid \\
& =\left|\int_{0}^{1} \frac{d}{d r}(F(|t|, x+r t)+F(|t|, x-r t)) d r\right| \\
& =\left|\sum_{j=1}^{n} t_{j} \int_{0}^{1}\left(F_{j}(|t|, x+r t)-F_{j}(|t|, x-r t)\right) d r\right| \leq C|t|^{1+\alpha} .
\end{aligned}
$$

Therefore, $|F(0, x+t)+F(0, x-t)-2 F(0, x)| \leq C|t|^{1+\alpha}$ and the proposition is proved.

Proposition 2. If the inequality

$$
\left|f\left(x_{0}, x\right)-f\left(x_{0}^{\prime}, x^{\prime}\right)\right| \leq C x_{0}^{-\delta+\alpha}\left|\left(x_{0}, x\right)-\left(x_{0}^{\prime}, x^{\prime}\right)\right|^{\delta}
$$

holds whenever $x_{0} / 2 \leq x_{0}^{\prime} \leq 2 x_{0}$ and $\left|x-x^{\prime}\right| \leq 2 x_{0}$, then $f$ belongs to $B_{\delta}^{+}(\alpha)$. (In other words, we need only check the bound on $f$ in each Whitney cube of the upper half space.) 
P roof. Suppose first that $x_{0} / 2 \leq x_{0}^{\prime} \leq 2 x_{0}$. We want to show that the restriction $\left|x-x^{\prime}\right| \leq 2 x_{0}$ can be dropped. Let $2^{N-1} x_{0} \leq\left|x-x^{\prime}\right| \leq 2^{N} x_{0}$, $N \geq 1$. Define the sequence of points $z^{0}=\left(x_{0}, x\right), z^{1}=\left(2 x_{0}, x\right), \ldots, z^{N}=$ $\left(2^{N} x_{0}, x\right), z^{N+1}=\left(2^{N} x_{0}, x^{\prime}\right), z^{N+2}=\left(2^{N-1} x_{0}, x^{\prime}\right), \ldots, z^{2 N+1}=\left(x_{0}, x^{\prime}\right)$, and $z^{2 N+2}=\left(x_{0}^{\prime}, x^{\prime}\right)$. Then

$$
\left|f\left(x_{0}, x\right)-f\left(x_{0}^{\prime}, x^{\prime}\right)\right| \leq \sum_{k=0}^{2 N+1}\left|f\left(z^{k}\right)-f\left(z^{k+1}\right)\right|,
$$

and the sum is majorized by the series $\sum_{k=0}^{N}\left(2^{k} x_{0}\right)^{\alpha} \leq \max \left(2^{N \alpha}, 1\right) x_{0}^{\alpha} \leq$ $x_{0}^{-\delta+\alpha}\left|x-x^{\prime}\right|^{\delta}$ since $\alpha<\delta$.

We may now assume

$$
\left|f\left(x_{0}, x\right)-f\left(x_{0}^{\prime}, x^{\prime}\right)\right| \leq C x_{0}^{-\delta+\alpha}\left|\left(x_{0}, x\right)-\left(x_{0}^{\prime}, x^{\prime}\right)\right|^{\delta}
$$

holds whenever $x_{0} / 2 \leq x_{0}^{\prime} \leq 2 x_{0}$. To check the inequality in general we may as well assume that $x_{0}^{\prime} \leq x_{0} / 2$. Let $x_{k+1}=x_{k} / 2, k=0,1, \ldots, N$. Choose $N$ so that $2^{-N} x_{0}>x_{0}^{\prime}$ and $2^{-N-1} x_{0} \leq x_{0}^{\prime}$. Our hypothesis implies $\left|f\left(x_{0}, x\right)-f\left(x_{0}^{\prime}, x^{\prime}\right)\right| \leq C x_{0}^{-\delta+\alpha}\left|x-x^{\prime}\right|^{\delta}, \quad\left|f\left(x_{k}, x^{\prime}\right)-f\left(x_{k+1}, x^{\prime}\right)\right| \leq C x_{k}^{\alpha}$. Also, $\left|f\left(x_{N}, x^{\prime}\right)-f\left(x_{0}^{\prime}, x^{\prime}\right)\right| \leq C\left(x_{0}^{\prime}\right)^{\alpha}$. Therefore,

$$
\begin{aligned}
\left|f\left(x_{0}, x\right)-f\left(x_{0}^{\prime}, x^{\prime}\right)\right| & \leq C\left(x_{0}^{-\delta+\alpha}\left|x-x^{\prime}\right|^{\delta}+\sum_{k=0}^{N} 2^{k \alpha}\left(x_{0}^{\prime}\right)^{\alpha}\right) \\
& \leq C\left(x_{0}^{-\delta+\alpha}\left|x-x^{\prime}\right|^{\delta}+\max \left(2^{N \alpha}, 1\right)\left(x_{0}^{\prime}\right)^{\alpha}\right) \\
& \leq C\left(x_{0}^{-\delta+\alpha}+\left(x_{0}^{\prime}\right)^{-\delta+\alpha}\right)\left|\left(x_{0}, x\right)-\left(x_{0}^{\prime}, x^{\prime}\right)\right|^{\delta}
\end{aligned}
$$

because $\left|x_{0}-x\right|^{\delta} \geq 2^{N \delta}\left(x_{0}^{\prime}\right)^{\delta} \geq \max \left(2^{N \alpha}, 1\right)\left(x_{0}^{\prime}\right)^{\delta}$.

Lemma 1. Let $w \in C_{0}^{\infty}(I),-1<\alpha-\delta<0, \Delta w(X)=g(X), X \in$ $\mathbf{R}_{+} \times \mathbf{R}^{n}$, and $\partial_{0} w(0, x)=0, x \in \mathbf{R}^{n}$. Then

$$
\left\|\nabla^{2} w\right\|_{B_{\delta}^{+}(\alpha)} \leq C\|g\|_{B_{\delta}^{+}(\alpha)} .
$$

Proof. Extend $g$ to all of $R^{n+1}$ as an even function of $x_{0}: g\left(x_{0}, x\right)=$ $g\left(-x_{0}, x\right)$. Then

$$
\begin{gathered}
w(X)=c_{n} \int_{\mathbf{R}^{n+1}}\left|X-X^{\prime}\right|^{1-n} g\left(X^{\prime}\right) d X^{\prime} \quad\left(\text { insert } \log \left|X-X^{\prime}\right| \text { if } n=1\right), \\
\frac{\partial^{2} w}{\partial x_{i} \partial x_{j}}(X)=\int_{\mathbf{R}^{n+1}} K\left(X-X^{\prime}\right) g\left(X^{\prime}\right) d X^{\prime}
\end{gathered}
$$

where $K$ is a standard Calderón-Zygmund kernel, homogeneous of degree $-(n+1)$, with mean value zero on the sphere. So the proof of this lemma 
amounts to proving that a standard Calderón-Zygmund operator is bounded on $B_{\delta}^{+}(\alpha)$ extended evenly to all of $R^{n+1}$.

Fix $X, Y \in R^{n+1}$. Suppose that $|X-Y|=\varepsilon$. Then

$$
w(X)-w(Y)=\int K\left(X-X^{\prime}\right) g\left(X^{\prime}\right) d X^{\prime}-\int K\left(Y-X^{\prime}\right) g\left(X^{\prime}\right) d X^{\prime} .
$$

Also,

The first claim is that

$$
\int K\left(X-X^{\prime}\right) g\left(X^{\prime}\right) d X^{\prime}=\int K\left(X^{\prime}\right) g\left(X-X^{\prime}\right) d X^{\prime} .
$$

$$
\left|\int_{\left|X^{\prime}\right| \leq 10 \varepsilon} K\left(X^{\prime}\right) g\left(X-X^{\prime}\right) d X^{\prime}\right| \leq C \varepsilon^{\delta}\left|x_{0}\right|^{\alpha-\delta} .
$$

Indeed,

$$
\begin{gathered}
\left|\int_{\left|X^{\prime}\right| \leq 10 \varepsilon} K\left(X^{\prime}\right) g\left(X-X^{\prime}\right) d X^{\prime}\right|=\left|\int_{\left|X^{\prime}\right| \leq 10 \varepsilon} K\left(X^{\prime}\right)\left(g\left(X-X^{\prime}\right)-g(X)\right) d X^{\prime}\right| \\
\leq C \int_{\left|X^{\prime}\right| \leq 10 \varepsilon}\left|X^{\prime}\right|^{-(n+1)}\left(\left|x_{0}-x_{0}^{\prime}\right|^{-\delta+\alpha}+\left|x_{0}\right|^{-\delta+\alpha}\right)\left|X^{\prime}\right|^{\delta} d X^{\prime} .
\end{gathered}
$$

This splits into three terms. One is

$$
\int_{\left|X^{\prime}\right| \leq 10 \varepsilon}\left|X^{\prime}\right|^{-(n+1)+\delta}\left|x_{0}\right|^{-\delta+\alpha} d X^{\prime}=C \varepsilon^{\delta}\left|x_{0}\right|^{-\delta+\alpha} .
$$

The second is

$$
\int_{\substack{\left|X^{\prime}\right| \leq 10 \varepsilon \\\left|x-x_{0}^{\prime}\right| \geq \frac{1}{2}\left|x_{0}\right|}}\left|X^{\prime}\right|^{-(n+1)+\delta}\left|x_{0}-x_{0}^{\prime}\right|^{-\delta+\alpha} d X^{\prime},
$$

which is controlled by the first because $\alpha-\delta<0$ implies $\left|x_{0}-x_{0}^{\prime}\right|^{-\delta+\alpha}<$ $\left(\frac{1}{2}\left|x_{0}\right|\right)^{-\delta+\alpha}$. Finally, the integral

$$
\int_{\substack{\left|X^{\prime}\right| \leq 10 \varepsilon \\\left|x_{0}-x_{0}^{\prime}\right|<\frac{1}{2}\left|x_{0}\right|}}\left|X^{\prime}\right|^{-(n+1)+\delta}\left|x_{0}-x_{0}^{\prime}\right|^{-\delta+\alpha} d X^{\prime}
$$

is zero unless $\left|x_{0}\right| \leq 20 \varepsilon$. Note also that $\left|x_{0}^{\prime}\right| \geq \frac{1}{2}\left|x_{0}\right|$, so that $\left|X^{\prime}\right| \geq$ $\frac{1}{4}\left|x^{\prime}\right|+\frac{1}{4}\left|x_{0}\right|$. Make the change of variables $t=x_{0}-x_{0}^{\prime}$. Then the integral is dominated by

$$
\begin{aligned}
& \int_{\left|x^{\prime}\right| \leq 20 \varepsilon} \int_{|t| \leq\left|x_{0}\right|}\left(\left|x^{\prime}\right|+\left|x_{0}\right|\right)^{-(n+1)+\delta}|t|^{-\delta+\alpha} d t d x^{\prime} \\
& \leq\left|x_{0}\right|^{1-\delta+\alpha} \int_{\left|x_{0}\right| \leq\left|x^{\prime}\right| \leq 20 \varepsilon}\left|x^{\prime}\right|^{-(n+1)+\delta} d x^{\prime} \leq\left|x_{0}\right|^{\alpha} \leq C \varepsilon^{\delta}\left|x_{0}\right|^{-\delta+\alpha} .
\end{aligned}
$$


This concludes the proof of the claim.

We now have

$$
\begin{aligned}
\left|\int_{\left|X^{\prime}\right| \leq 10 \varepsilon} K\left(X^{\prime}\right) g\left(X-X^{\prime}\right) d X+\int_{\left|X^{\prime}\right| \leq 10 \varepsilon} K\left(X^{\prime}\right) g\left(Y-X^{\prime}\right) d X^{\prime}\right| \\
\leq C \varepsilon^{\delta}\left(\left|x_{0}\right|^{\alpha-\delta}+\left|y_{0}\right|^{\alpha-\delta}\right) .
\end{aligned}
$$

Next we need to look at the rest of $w(X)-w(Y)$. Since $g\left(X^{\prime}\right)$ is supported in $I$, the remainder is

$$
\begin{aligned}
\int \bar{K}\left(X^{\prime}\right)\left(g\left(X-X^{\prime}\right)-\right. & \left.g\left(Y-X^{\prime}\right)\right) d X^{\prime} \\
& =\int\left(\bar{K}\left(X-X^{\prime}\right)-\bar{K}\left(Y-X^{\prime}\right)\right) g\left(X^{\prime}\right) d X^{\prime}
\end{aligned}
$$

where $\bar{K}\left(X^{\prime}\right)=K\left(X^{\prime}\right)$ for $10 \varepsilon<\left|X^{\prime}\right|<10$ and $\bar{K}\left(X^{\prime}\right)=0$ otherwise. It is easy to check that since $|X-Y|=\varepsilon,\left|\bar{K}\left(X-X^{\prime}\right)-\bar{K}\left(Y-X^{\prime}\right)\right| \leq$ $C \varepsilon /\left|X-X^{\prime}\right|^{n+2}$. Therefore,

$$
\begin{aligned}
& \left|\int\left(\bar{K}\left(X-X^{\prime}\right)-\bar{K}\left(Y-X^{\prime}\right)\right) g\left(X^{\prime}\right) d X^{\prime}\right| \\
& \quad=\left|\int\left(\bar{K}\left(X-X^{\prime}\right)-\bar{K}\left(Y-X^{\prime}\right)\right)\left(g\left(X^{\prime}\right)-g(X)\right) d X^{\prime}\right| \\
& \quad \lesssim \int_{1 \geq\left|X-X^{\prime}\right| \geq 9 \varepsilon} \frac{\varepsilon}{\left|X-X^{\prime}\right|^{n+2}}\left(\left|x_{0}^{\prime}\right|^{-\delta+\alpha}+\left|x_{0}\right|^{-\delta+\alpha}\right)\left|X-X^{\prime}\right|^{\delta} d X^{\prime} .
\end{aligned}
$$

This splits into two terms:

$$
\int_{\left|X-X^{\prime}\right| \geq 9 \varepsilon} \varepsilon\left|X-X^{\prime}\right|^{\delta-(n+2)} d X^{\prime}\left|x_{0}\right|^{-\delta+\alpha} \lesssim \varepsilon \cdot \varepsilon^{\delta-1} \cdot\left|x_{0}\right|^{-\delta+\alpha}=\varepsilon^{\delta}\left|x_{0}\right|^{-\delta+\alpha} .
$$

In the second integral

$$
\int_{\left|X-X^{\prime}\right| \geq 9 \varepsilon} \varepsilon\left|X-X^{\prime}\right|^{\delta-(n+2)}\left|x_{0}\right|^{-\delta+\alpha} d X^{\prime}
$$

we can restrict to the region $\left|x_{0}^{\prime}\right|<\frac{1}{2}\left|x_{0}\right|$ because otherwise the integral is dominated by the previous one. But $\left|x_{0}^{\prime}\right|<\frac{1}{2}\left|x_{0}\right|$ implies $\left|x_{0}-x_{0}^{\prime}\right|>\frac{1}{2}\left|x_{0}\right|$. Thus $\left|X-X^{\prime}\right| \geq \frac{1}{4}\left|x-x^{\prime}\right|+\frac{1}{4}\left|x_{0}\right|$, and this last integral is dominated by

$$
\begin{aligned}
\int_{\substack{\left|X-X^{\prime}\right| \geq 9 \varepsilon \\
\left|x_{0}^{\prime}\right| \leq \frac{1}{2}\left|x_{0}\right|}} & \varepsilon\left(\left|x-x^{\prime}\right|+\left|x_{0}\right|\right)^{\delta-(n+2)}\left|x_{0}^{\prime}\right|^{-\delta+\alpha} d x^{\prime} d x_{0}^{\prime} \\
& \lesssim \varepsilon\left|x_{0}\right|^{1-\delta+\alpha} \int_{|z| \geq \varepsilon+\left|x_{0}\right|}|z|^{\delta-(n+2)} d z \\
& =\varepsilon\left|x_{0}\right|^{1-\delta+\alpha}\left(\varepsilon+\left|x_{0}\right|\right)^{\delta-2} \leq \varepsilon^{\delta}\left|x_{0}\right|^{-\delta+\alpha}, \quad \text { where } z \in \mathbf{R}^{n} .
\end{aligned}
$$

This concludes Lemma 1. 
Lemma 2. If $w \in C_{0}^{\infty}(I),-1<\alpha-\delta<0$,

$$
\begin{cases}\Delta w=0, & y_{0}>0, \\ \partial_{0} w=F, & y_{0}=0,\end{cases}
$$

then $\left\|w_{i j}\right\|_{B_{b}^{+}(\alpha)} \leq C\|F\|_{\Lambda(\alpha+1)}$.

Proof. We have

$$
\begin{gathered}
\hat{w}\left(x_{0}, \xi\right)=\int_{R^{n}} e^{-i x \cdot \xi} w\left(x_{0}, x\right) d x=e^{-x_{0}|\xi|}(-1 /|\xi|) \hat{F}(\xi), \\
w\left(x_{0}, x\right)=P_{x_{0}} * g(x) \quad \text { and } g \in \Lambda(\alpha+2) .
\end{gathered}
$$

Thus by standard arguments [19]

$$
\left|\nabla w_{i j}\right| \leq C\left|x_{0}\right|^{\alpha-1}\|g\|_{\Lambda(\alpha+2)} \leq C\left|x_{0}\right|^{\alpha-1}\|f\|_{\Lambda(\alpha+1)} .
$$

Therefore, if $x_{0} / 2 \leq x_{0}^{\prime} \leq 2 x_{0}$,

$$
\left|w_{i j}(X)-w_{i j}\left(X^{\prime}\right)\right|=\left|\int_{0}^{1}\left[\frac{d}{d t} w_{i j}\left(X^{\prime}+t\left(X^{\prime}-X\right)\right)\right] d t\right| \leq C x_{0}^{\alpha-1}\left|X-X^{\prime}\right|
$$

If we restrict to $\left|X-X^{\prime}\right| \leq 10 x_{0}$, then this is majorized by $x_{0}^{\alpha-\delta}\left|X-X^{\prime}\right|^{\delta}$. Now Lemma 2 follows from Proposition 2.

Define $X=\sum_{j=0}^{n} b_{j} \partial_{j}+b$ for $b_{j} \in \mathbf{R}, b \in \mathbf{R}, b_{0} \neq 0$.

LeMmA 3. If $w \in C_{0}^{\infty}(I),-1<\alpha-\delta<0, \alpha<0$,

$$
\begin{cases}\Delta w=g, & y_{0}>0 \\ X w=f, & y_{0}=0\end{cases}
$$

then $\left\|w_{i j}\right\|_{B_{b}^{+}(\alpha)} \leq C\left(\|g\|_{B_{\delta}^{+}(\alpha)}+\|f\|_{A(\alpha+1)}\right)$.

P roof. Set $F(y)=\partial_{0} w(0, y)$. We have

$$
\hat{w}\left(y_{0}, \xi\right)=\int_{\mathbf{R}^{n}} e^{-i y \cdot \xi} w\left(y_{0}, y\right) d y, \quad \hat{F}(\xi)=\int_{\mathbf{R}^{n}} e^{-i y \cdot \xi} F(y) d y .
$$

From Lemmas 1 and 2 we can solve

$$
\left\{\begin{array} { l l } 
{ \Delta w ^ { 0 } = g , } & { y _ { 0 } > 0 , } \\
{ \partial _ { 0 } w ^ { 0 } = 0 , } & { y _ { 0 } = 0 , }
\end{array} \quad \text { and } \quad \left\{\begin{array}{ll}
\Delta w^{1}=0, & y_{0}>0 \\
\partial_{0} w^{1}=F, & y_{0}=0
\end{array}\right.\right.
$$

with $\left\|w_{i j}^{0}\right\|_{B_{\delta}^{+}(\alpha)} \leq C\|g\|_{B_{\delta}^{+}(\alpha)}$ and $\left\|w_{i j}^{1}\right\|_{B_{\delta}^{+}(\alpha)} \leq C\|F\|_{\Lambda(\alpha+1)}$. Then $w=$ $w^{0}+w^{1}$. We need only show that $\|F\|_{A(\alpha+1)}$ is majorized by $\|g\|_{B_{\delta}^{+}(\alpha)}+$ $\|f\|_{\Lambda(\alpha+1)}$. Indeed, $f=X w=X w^{0}+X w^{1}$ implies $\left\|X w^{1}\right\|_{\Lambda(\alpha+1)} \leq$ $\left\|X w^{0}\right\|_{\Lambda(\alpha+1)}+\|f\|_{\Lambda(\alpha+1)}$. But by Proposition 1 and Lemma 2

$$
\left\|X w^{0}\right\|_{\Lambda(\alpha+1)} \leq C\left\|\nabla^{2} w^{0}\right\|_{B_{\delta}^{+}(\alpha)} \leq C\|g\|_{B_{\delta}^{+}(\alpha)} .
$$


Thus $\left\|X w^{1}\right\|_{A(\alpha+1)} \leq C\left(\|g\|_{B_{\delta}^{+}(\alpha)}+\|f\|_{A(\alpha+1)}\right)$. Next, $\left(w^{1}\right)^{\wedge}(r, \xi)=$ $-e^{-r|\xi|}(-1 /|\xi|) \hat{F}(\xi)$, so that

$$
\left(X w^{1}\right)^{\wedge}(0, \xi)=b_{0} \hat{F}(\xi)-\left(\sum_{k=1}^{n} i b_{j} \xi_{j} /|\xi|+b /|\xi|\right) \hat{F}(\xi) .
$$

Because $b_{0} \neq 0$, we can rewrite this as

$$
\hat{F}(\xi)=\left(b_{0}-\sum_{j=1}^{n} i b_{j} \xi_{j} /|\xi|-b /|\xi|\right)^{-1}\left(X w^{1}\right)^{\wedge}(0, \xi) .
$$

Hence by standard multiplier theory

$$
\|F\|_{\Lambda(\alpha+1)} \leq C\left\|X w^{1}\right\|_{\Lambda(\alpha+1)}
$$

This proves Lemma 3.

Proof of Theorem A. It suffices to estimate $w$ in a small neighborhood $U$ of the origin. If we make the change of variables $\tilde{w}(Y)=w(\theta Y)$ with $|Y| \leq 1$, we observe that

$$
\left|b_{j}(\theta Y)-b_{j}(0)\right| \leq C \theta^{s}
$$

and $\left|b_{j}(\theta Y)-b_{j}\left(\theta Y^{\prime}\right)\right| \leq C \theta^{s}\left|Y-Y^{\prime}\right|^{s}$ for all $|Y| \leq 1,\left|Y^{\prime}\right| \leq 1$.

Taking $\theta$ sufficiently small, but fixed, we see that $b_{j}(\theta Y)-b_{j}(0)$ has arbitrarily small $\Lambda(s)$ norm. Similarly, $a_{i j}(\theta Y)-a_{i j}(0)$ has small $\Lambda^{+}(s)$ norm. After a linear change of variable we can replace $a_{i j}(0)$ by the identity matrix $\delta_{i j}$. Thus we reduce to the special case in which

$$
\Delta w(Y)+\sum_{i, j=0}^{n} \varepsilon_{i j}(Y) w_{i j}(Y)=R(Y), \quad y_{0}>0, \quad|Y| \leq 2,
$$

$$
X w(0, y)+\sum_{j=0}^{n} \beta_{j}(y) w_{j}(0, y)=R(y), \quad|y| \leq 2,
$$

with

$$
\begin{gathered}
X=\sum_{j=0}^{n} b_{j} \partial / \partial y_{j}, \quad b_{j} \in \mathbf{R}, \quad b_{0} \geq c_{0}>0, \\
\left\|\beta_{j}\right\|_{\Lambda(s)} \leq \varepsilon, \quad\left\|\varepsilon_{i j}\right\|_{\Lambda^{+}(s)} \leq \varepsilon,
\end{gathered}
$$

for some $\varepsilon$ as small as we like. We can also replace $w$ by $\psi w$, where $\psi \in$ $C_{0}^{\infty}\left(\frac{3}{2} I\right), \psi(y)=1$ for $y \in I$. This introduces error terms on the right hand side of $(*)$ of the form $\psi_{i} w_{i}, \psi_{i j} w$. It introduces a term $-(X \psi) w-$ $\sum \beta_{j}\left(\partial_{j} \psi\right) w$ on the right hand side of $(* *)$. Since norms of these expressions appear on the right hand side in the conclusion of Theorem $A$, we may add these terms to the right hand side. Thus we are reduced to the special case in which $\operatorname{supp} w \subset 2 I$. We can also assume that $a_{i j}$ and $b_{j}$ are supported 
in $2 I$. Set $\Delta w=g_{1}$ and $X w(0, x)=g_{0}(x)$. There is an explicit operator $K_{1}$ solving the boundary value problem of Lemma 3: $w=K_{1}\left(g_{0}, g_{1}\right)$. Lemma 3 gives us the estimates

$$
\left\|\nabla^{2} K_{1}\left(g_{0}, g_{1}\right)\right\|_{B_{\delta}^{+}(s-1)} \leq C\left(\left\|g_{0}\right\|_{A(s)}+\left\|g_{1}\right\|_{B_{\delta}^{+}(s-1)}\right) .
$$

Denote by $K_{0}\left(g_{0}, g_{1}\right)$ the restriction of $w$ to $x_{0}=0$. Then by Proposition 1 ,

$$
\left\|\nabla K_{0}\left(g_{0}, g_{1}\right)\right\|_{\Lambda(s)} \leq C\left(\left\|g_{0}\right\|_{\Lambda(s)}+\left\|g_{1}\right\|_{B_{\delta}^{+}(s-1)}\right) .
$$

The equations for $w$ imply

$$
g_{0}+\beta_{j} \partial_{j} K_{0}\left(g_{0}, g_{1}\right)=f, \quad g_{1}+\varepsilon_{i j} \partial_{i j} K_{1}\left(g_{0}, g_{1}\right)=R .
$$

This is a single equation on the space $\Lambda(s) \times B_{\delta}^{+}(s-1)$ of the form (id + $T)\left[\begin{array}{l}g_{0} \\ g_{1}\end{array}\right]=\left[\begin{array}{l}f \\ R\end{array}\right]$, where $T$ is a contraction. Hence $(\mathrm{id}+T)^{-1}$ is bounded and

$$
\left\|g_{0}\right\|_{A(s)}+\left\|g_{1}\right\|_{B_{\delta}^{+}(s-1)} \leq C\left(\|f\|_{\Lambda(s)}+\|R\|_{B_{\delta}^{+}(s-1)}\right) .
$$

This concludes the proof of Theorem A.

We need one more linear estimate, namely the well-known estimates for the Dirichlet problem in ordinary Hölder classes (see [7, Theorem 6.19]).

Theorem B. Suppose that

$$
\begin{gathered}
\sum_{i, j=0}^{n} a_{i j}(Y) w_{i j}(Y)=R(Y), \quad Y \in \mathbf{R}_{+} \times \mathbf{R}^{n}, \\
w(0, y)=f(y), \quad y \in \mathbf{R}^{n} .
\end{gathered}
$$

Then

$$
\begin{aligned}
\left\|\nabla^{2} w\right\|_{\Lambda^{+}(\beta, I)} \leq & C\left(\|R\|_{\Lambda^{+}(\beta, 2 I)}+\|f\|_{\Lambda^{+}(\beta+2,2 I)}\right. \\
& \left.+\|\nabla w\|_{\Lambda^{+}(\beta, 2 I)}+\|w\|_{\Lambda^{+}(\beta, 2 I)}\right) .
\end{aligned}
$$

The constant $C$ depends only on the $\Lambda^{+}(\beta)$ norm of $a_{i j}$ and the ellipticity constant.

4. Nonlinear estimates. We are now prepared to prove Theorem 1. We begin with a Lipschitz function $\varphi$ and suppose that $\log h(\varphi(x), x) \in \Lambda(\alpha)$ for some $\alpha>0$. By Caffarelli's theorem (Theorem 3), we know that $u \in C^{1, s}$ for some $s>0$. Following Kinderlehrer and Nirenberg, we change variables to $y_{j}=x_{j}, j=1, \ldots, n, y_{0}=u$. The inverse function theorem implies that we can write $x_{0}=\psi\left(y_{0}, y_{1}, \ldots, y_{n}\right)$ for a function $\psi \in C^{1, s}\left([0, \infty) \times \mathbf{R}^{n}\right) \cap$ $C^{\infty}\left((0, \infty) \times \mathbf{R}^{n}\right)$. We can rewrite the equations for $u$ in terms of equations 
for $\psi$ :

$$
\begin{aligned}
\frac{-1}{\psi_{0}} \sum_{j=1}^{n} \psi_{j j}+\frac{2}{\psi_{0}^{2}} \sum_{j=1}^{n} \psi_{j} \psi_{j 0}-\frac{1+\sum_{j=1}^{n} \psi_{j}^{2}}{\psi_{0}^{3}} \psi_{00}=0 & \text { for } y_{0}>0 \\
\left(1+\sum_{j=1}^{n} \psi_{j}^{2}\right)^{1 / 2} / \psi_{0}=\varphi\left(\psi, y_{1}, \ldots, y_{n}\right) & \text { for } y_{0}=0
\end{aligned}
$$

(Here $\psi_{j}=\partial \psi / \partial y_{j}$ and $\psi_{i j}=\partial^{2} \psi / \partial y_{i} \partial y_{j}, i, j=0, \ldots, n$.) It is easy to check that these equations take the form

$$
\sum_{i, j=0}^{n} a_{i j}(\nabla \psi) \psi_{i j}=0, \quad g\left(\psi_{0}, \psi_{1}, \ldots, \psi_{n}\right)=\varphi\left(\psi, y_{1}, \ldots, y_{n}\right)
$$

with $a_{i j}$ uniformly elliptic and $C^{\infty}, g \in C^{\infty}$. Because $c \leq \psi_{0} \leq C$ on the boundary, we also have $-c^{\prime} \leq \partial g / \partial \psi_{0} \leq-C^{\prime}<0$.

Let $t \in \mathbf{R}^{n}$ and consider $w\left(x_{0}, x\right)=\psi\left(x_{0}, x+t\right)-\psi\left(x_{0}, x\right)$. Define $\psi^{t}\left(x_{0}, x\right)=\psi\left(x_{0}, x+t\right)$. Subtracting the equations for $\psi$ and $\psi^{t}$ we find

$$
\sum_{i, j=0}^{n} a_{i j}(\nabla \psi) w_{i j}=\sum_{i, j=0}^{n}\left(a_{i j}\left(\nabla \psi^{t}\right)-a_{i j}(\nabla \psi)\right) \psi_{i j}^{t}
$$

Also,

$$
\begin{aligned}
\int_{0}^{1} \frac{d}{d s} g\left(\nabla \psi+s\left(\nabla \psi^{t}-\nabla \psi\right)\right) d s & =g\left(\nabla \psi^{t}\right)-g(\nabla \psi) \\
& =\varphi\left(\psi^{t}, y+t\right)-\varphi(\psi, y)
\end{aligned}
$$

Hence,

$$
\sum_{j=0}^{n}\left[\int_{0}^{1} \frac{\partial g}{\partial \psi_{j}}\left(\nabla \psi+s\left(\nabla \psi^{t}-\nabla \psi\right)\right) d s\right] w_{j}=f
$$

where $f=\varphi\left(\psi^{t}, y+t\right)-\varphi(\psi, y)$. We can rewrite these equations as

$$
\begin{aligned}
\sum_{i, j=0}^{n} a_{i j}(\nabla \psi) w_{i j} & =R, \quad y_{0}>0 \\
\sum_{j=0}^{n} b_{j} w_{j} & =f, \quad y_{0}=0
\end{aligned}
$$


where

$$
\begin{aligned}
R & =\sum_{i, j=0}^{n}\left(a_{i j}\left(\nabla \psi^{t}\right)-a_{i j}(\nabla \psi)\right) \psi_{i j}^{t} \\
f(y) & =\varphi\left(\psi^{t}, y+t\right)-\varphi(\psi, y) \\
b_{j}(y) & =\int_{0}^{1} \frac{\partial g}{\partial \psi_{j}}\left(\nabla \psi(0, y)+s\left(\nabla \psi^{t}(0, y)-\nabla \psi(0, y)\right)\right) d s
\end{aligned}
$$

Indeed,

$$
\begin{gathered}
\int_{0}^{1} \frac{d}{d s} g\left(\nabla \psi+s\left(\nabla \psi^{t}-\nabla \psi\right)\right) d s=g\left(\nabla \psi^{t}\right)-g(\nabla \psi)=f \\
\frac{d}{d s} g\left(\nabla \psi+s\left(\nabla \psi^{t}-\nabla \psi\right)\right)=\sum_{j=0}^{n} \frac{\partial g}{\partial \psi_{j}}\left(\nabla \psi+s\left(\nabla \psi^{t}-\nabla \psi\right)\right) w_{j}
\end{gathered}
$$

so that $b_{j}$ has the desired form.

Because $\psi$ and $\psi^{t}$ belong to $\Lambda^{+}(s+1)$, we see that $b_{j} \in \Lambda(s)$ and $a_{i j}(\nabla \psi) \in \Lambda^{+}(s)$. Furthermore, it is easy to check from bounds on $u$ in Whitney cubes that $\psi_{i j}^{t} \in B_{\delta}^{+}(s-1)$ whenever $\delta<s$ (with bound independent of $t$ ). Next,

$$
\begin{aligned}
a_{i j}\left(\nabla \psi^{t}\right)-a_{i j}(\nabla \psi) & =\int_{0}^{1} \frac{d}{d s} a_{i j}\left(\nabla \psi+s\left(\nabla \psi^{t}-\nabla \psi\right)\right) d s \\
& =\sum_{k=0}^{n} \int_{0}^{1} \frac{\partial a_{i j}}{\partial \psi_{k}}\left(\nabla \psi+s\left(\nabla \psi^{t}-\nabla \psi\right)\right) d s\left(\psi_{k}^{t}-\psi_{k}\right) \\
& =\sum_{k=0}^{n} c_{i j}^{k}\left(\psi_{k}^{t}-\psi_{k}\right), \text { where } c_{i j}^{k} \in \Lambda^{+}(s) .
\end{aligned}
$$

Remark. For any $v \in \Lambda^{+}(s), 0<\delta<s<1$, $\left\|v^{t}-v\right\|_{\Lambda^{+}(s)} \leq$ $2\|v\|_{\Lambda^{+}(s)}|t|^{s-\delta}$.

Proof. We have

$$
\left|\left(v^{t}(Y)-v(Y)\right)-\left(v^{t}\left(Y^{\prime}\right)-v\left(Y^{\prime}\right)\right)\right| \leq 2|t|^{s}\|v\|_{\Lambda+(s)} .
$$

Also,

$$
\begin{aligned}
\left|\left(v^{t}(Y)-v(Y)\right)-\left(v^{t}\left(Y^{\prime}\right)-v\left(Y^{\prime}\right)\right)\right| & =\left|\left(v^{t}(Y)-v^{t}\left(Y^{\prime}\right)\right)-\left(v(Y)-v\left(Y^{\prime}\right)\right)\right| \\
& \leq 2\left|Y-Y^{\prime}\right|^{s}\|v\|_{\Lambda^{+}(s)} .
\end{aligned}
$$

But $\min \left(|t|^{s},\left|Y-Y^{\prime}\right|^{s}\right) \leq|t|^{s-\delta}\left|Y-Y^{\prime}\right|^{\delta}$. So

$$
\left|\left(v^{t}-v\right)(Y)-\left(v^{t}-v\right)\left(Y^{\prime}\right)\right| \leq 2|t|^{s-\delta}\left|Y-Y^{\prime}\right|^{\delta}\|v\|_{\Lambda^{+}(s)} .
$$


Because $\psi_{k} \in \Lambda^{+}(s)$ we can conclude $\left\|\psi_{k}^{t}-\psi_{k}\right\|_{\Lambda^{+}(s)} \leq C|t|^{s-\delta}$, and hence

$$
\left\|a_{i j}(\nabla \psi)\right\|_{\Lambda^{+}(s)} \leq C|t|^{s-\delta} .
$$

Finally, we have

$$
\|R\|_{B_{\delta}^{+}(s-1)} \leq C|t|^{s-\delta} .
$$

A similar discussion with $f$ shows that $\|f\|_{\Lambda(s)} \leq C|t|^{\alpha-s}$. (We may evidently assume $s<\alpha$ since otherwise there is nothing to prove. When $\alpha \geq 1$, we need to take second differences: $w=\psi^{t}+\psi^{-t}-2 \psi$, but the other aspects of the proof barely change.)

We are nearly in a position to apply Theorem A. However, we must also estimate $w_{i}$ and $w$ in the interior. In fact, we have just shown $\left\|w_{k}\right\|_{A^{+}(s)} \leq$ $C|t|^{s-\delta}$. But it is obvious that $\left\|w_{k}\right\|_{B_{b}^{+}(s-1)} \leq C\left\|w_{k}\right\|_{\Lambda^{+}(s)}$. The estimate for $w$ is even simpler. Therefore, applying Theorem $A$, we find that

$$
\left\|w_{i j}\right\|_{B_{\delta}^{+}(s-1)} \leq C\left(|t|^{s-\delta}+|t|^{\alpha-s}\right)\left\|w_{k}\right\|_{\Lambda^{+}(s)} \text {. }
$$

In particular, if we restrict to the space $I \cap\left\{y_{0} \geq h\right\}$, we find

$$
\begin{aligned}
\mid \psi_{i j}(h, x+t)-\psi_{i j}(h, x)-\left(\psi_{i j}(\right. & \left.\left.h, x^{\prime}+t\right)-\psi_{i j}\left(h, x^{\prime}\right)\right) \mid \\
& \leq C h^{s-1-\delta}\left(|t|^{s-\delta}+|t|^{\alpha-s}\right)\left|x-x^{\prime}\right|^{\delta} .
\end{aligned}
$$

Set $x^{\prime}=x-t$; then we have

$$
\left|\psi_{i j}(h, x+t)+\psi_{i j}(h, x-t)-2 \psi_{i j}(h, x)\right| \leq C h^{s-1-\delta}\left(|t|^{s}+|t|^{\alpha-s+\delta}\right) .
$$

This is the second difference we referred to in the introduction. Let $f(x)=$ $\psi(h, x)$. Then using the second difference norm for $\Lambda(\beta)$ we see that $\|f\|_{\Lambda(\beta+2)}$ $\leq C h^{s-1-\delta}$, where $\beta=\min (s, \alpha-s+\delta)$. Thus $\psi$ satisfies an equation of the type in Theorem $\mathrm{B}$ in the half space $y_{0} \geq h$. We already know that $\psi \in \Lambda^{+}(s+1)$, so that $\nabla \psi \in \Lambda^{+}(s)$ and $\psi \in \Lambda^{+}(s)$ with uniform bounds independent of $h$. (This is even better than we need, since $s-1-\delta<$ 0.) Finally, $\psi$ satisfies a homogeneous equation with coefficients $a_{i j}(\nabla \psi) \in$ $\Lambda^{+}(s)$ with uniform bounds independent of $h$. Therefore,

$$
\left\|\nabla^{2} \psi\right\|_{\Lambda+\left(\beta, I \cap\left\{y_{0} \geq h\right\}\right)} \leq C h^{s-1-\delta} .
$$

We conclude that on a Whitney cube $2 h \geq y_{0} \geq h,\left|Y-Y^{\prime}\right|<h / 2, Y=$ $\left(y_{0}, y\right), Y^{\prime}=\left(y_{0}^{\prime}, y^{\prime}\right)$,

$$
\left|\nabla^{2} \psi(Y)-\nabla^{2} \psi\left(Y^{\prime}\right)\right| \leq C h^{s-1-\delta}\left|Y-Y^{\prime}\right|^{\beta} \leq C h^{s-1-2 \delta+\beta}\left|Y-Y^{\prime}\right|^{\delta} .
$$

Therefore by Proposition $2, \nabla^{2} \psi \in B_{\delta}^{+}(s-1-\delta+\beta)$, and by Proposition 1 , $\nabla \psi \in \Lambda^{+}(s-\delta+\beta)$. Now if $s<\alpha-s+\delta$, then $s-\delta+\beta=2 s-\delta$. We now repeat the argument above with $s$ replaced by $2 s-\delta$. Thus we can essentially double the regularity $s$ at each step. We are constrained 
by $s<1$. If $\alpha<1+\delta$ we can always increase $s$ until $s>\alpha-s+\delta$. At that point $s-\delta+\beta=s-\delta+(\alpha-s+\delta)=\alpha$, and we have proved $\nabla \psi \in \Lambda^{+}(\alpha)$. In fact, by changing $\delta$, we can prove regularity in this way when $0<\alpha<2$. However, having obtained regularity for $0<\alpha<1+\delta$ one can also differentiate the equation any number of times to obtain Theorem 1 for any $\alpha>0$.

5. Continuous data. We will now prove Theorem 2. We are supposing that $\log h(\varphi(x), x)$ is continuous, so that by Caffarelli's theorem, $\varphi \in \lambda_{*}$. Define $k_{X}(Q)=N_{Q} \cdot \nabla G_{X}(Q)$, where $G_{X}(Y)$ is Green's function for $\Omega$ with pole at $X$. In the case $\varphi(x) \equiv a \cdot x+b, a \in \mathbf{R}^{n}, b \in \mathbf{R}, k_{X}(Q)$ is the ordinary Poisson kernel in a half space. In general, $\varphi \in \lambda_{*}$ implies that for any $\varepsilon>0$ and $M<\infty$, there exists $r_{0}>0$ such that for each $r \leq r_{0}$, there exist $a \in \mathbf{R}^{n}$ and $b \in \mathbf{R}$ such that

$$
|\varphi(x)-a \cdot x-b| \leq \varepsilon r / M \text { for }|x| \leq M r .
$$

The idea of the proof is to make use of the Rellich formula [10, Lemma 1.4]:

$$
\frac{1}{\omega_{n}} \int_{\partial \Omega} k_{X}(Q)^{2}\left\langle Q-X, N_{Q}\right\rangle d \sigma(Q)=\int_{\partial \Omega} k_{X}(Q) \frac{d \sigma(Q)}{|X-Q|^{n-1}},
$$

for any $X \in \Omega$, where $d \sigma$ denotes surface measure on $\partial \Omega$ and $\omega_{n}$ the surface area of the unit sphere in $\mathbf{R}^{n+1}$. The function $k_{X}(Q)$ will be very close to the ordinary Poisson kernel, and this will lead to control of $d \sigma(Q)=$ $\sqrt{1+|\nabla \varphi(x)|^{2}} d x$ which will lead in turn to control of $|\nabla \varphi(x)-a|$.

Without loss of generality we may suppose that $b=0$. We then have

$$
|\varphi(x)-a \cdot x| \leq \varepsilon r / M \text { for }|x| \leq M r .
$$

Throughout the argument $\varepsilon, r$, and $M$ will be fixed, but our bounds will be independent of these parameters. Moreover, we are doing analysis in a neighborhood of $x=0$, but the same exact argument works in $\left|x-x_{0}\right| \leq M r$. Note that since $\varphi$ if Lipschitz, $|\nabla \varphi(x)| \leq A$. We can also assume $|a| \leq A$. Our bounds will depend on the constant $A$.

Let $\alpha=\sqrt{1+|a|^{2}}$ and $N_{a}=(-1, a) / \alpha$, the outer unit normal to the half space $x_{0}>a \cdot x$. Let $P z=(a \cdot z) a /|a|^{2}$, the projection $\mathbf{R}^{n} \rightarrow \mathbf{R} a$. Define $x_{a}: \mathbf{R}^{n} \rightarrow \mathbf{R}^{n}$ by

$$
x_{a}(z)=(\mathrm{id}-P) z+P z / \alpha .
$$

It is easy to check that the mapping $Q_{a}: \mathbf{R}^{n} \rightarrow \mathbf{R}^{n+1}$ given by

$$
Q_{a}(z)=\left(a \cdot x_{a}(z), x_{a}(z)\right)=\left(a \cdot P z / \alpha, x_{a}(z)\right)
$$

is a linear isometry from $\mathrm{R}^{n}$ to the hyperplane $x_{0}=a \cdot x$ normal to $N_{a}$. 
Also, $d x=(1 / \alpha) d z$ and

$$
d \sigma(Q)=\sqrt{1+(\nabla \varphi(x))^{2}} d x=\sqrt{1+\left|\nabla \varphi\left(x_{a}(z)\right)\right|^{2}} d z / \alpha
$$

with the parametrization $x=x_{a}(z)$ and

$$
Q=(\varphi(x), x)=\left(\varphi\left(x_{a}(z)\right), x_{a}(z)\right) \equiv Q(z) .
$$

Let $P_{r}(z)=c_{n} r /\left(r^{2}+|z|^{2}\right)^{n+1}$ be the Poisson kernel for the upper half space $r>0$.

Lemma 4. For any $\varepsilon>0$ and any $M<\infty$ there exists $r_{0}>0$ depending only on the control in $\lambda_{*}$ of $\varphi$ and the modulus of continuity at $(\varphi(0), 0)$ of $h$ such that

$$
(1-\varepsilon / M) P_{r}(z) \leq k_{-r N_{a}}(Q(z)) \leq(1+\varepsilon / M) P_{r}(z)
$$

with $Q(z)=\left(\varphi\left(x_{a}(z)\right), x_{a}(z)\right)$ for all $|z| \leq M r$.

Proof. This lemma is a nearly immediate consequence of $[10$, Lemma 2.10], which says that if we define $K(X, Q)=k_{X}(Q) / h(Q)$, then for all $\varepsilon>0, M$ we can find $r_{0}>0$ such that for all $|z| \leq M r$,

$$
\begin{aligned}
(1-\varepsilon / M) h * \chi_{r}(Z) & \leq P_{r}(Z) / K\left(-r N_{a}, Q(Z)\right) \\
& \leq(1+\varepsilon / M) h * \chi_{r}(z)
\end{aligned}
$$

where $h=h(Q(z)), \chi_{r}(z)=r^{-n} \chi\left(r^{-1} z\right)$ with $\chi$ the characteristic function of the ball with volume $1\left(\int \chi(z) d z=1\right)$ and * denoting ordinary convolution on $\mathbf{R}^{n}$. While that lemma is stated for $C^{1}$ functions $\varphi$, it applies without change to functions $\varphi$ in $\lambda_{*}$. Next, observe that for $r_{0}$ sufficiently small, $r \leq r_{0}, h(Q(z))=(1+O(\varepsilon / M)) h_{0}$ for $|z| \leq M r$, where $h_{0}=h(Q(0))=h(\varphi(0), 0)$. Hence $h * \chi_{r}(z)=(1+O(\varepsilon / M)) h_{0}$ and Lemma 4 follows.

We are going to make use of $(\dagger)$ with $X=-r N_{a}$. Let $\psi \in C_{0}^{\infty}\left(B_{1}\right)$ be such that $0 \leq \psi \leq 1$ on $B_{1}$ and $\psi=1$ on $B_{1 / 2}$.

Lemma 5. For any $\delta>0$, there exists $M<\infty$ such that for all $\varepsilon>0$ there exists $r_{0}>0$ such that for $r \leq r_{0}$, and $X=-r N_{a}$,

$$
\begin{aligned}
\frac{1}{\omega_{n}} \int_{\partial \Omega} k_{X}(Q)^{2}\langle Q- & \left.X, N_{Q}\right\rangle d \sigma(Q) \\
& =\frac{1}{\omega_{n}} \int_{\partial \Omega} \psi(z / M r) P_{r}(z)^{2} r d z(1+O(\delta+\varepsilon)),
\end{aligned}
$$

$$
\begin{array}{r}
\int_{\partial \Omega} k_{X}(Q) \frac{d \sigma(Q)}{|X-Q|^{n-1}}=\int_{\mathbf{R}^{n}} \psi(z / M r) P_{r}(z)\left(|z|^{2}+r^{2}\right)^{(1-n) / 2} \\
\times \sqrt{1+\left|\nabla \varphi\left(x_{a}(z)\right)\right|^{2}} d z / \alpha,
\end{array}
$$


(c) $\frac{1}{\omega_{n}} \int_{\mathrm{R}^{n}} \psi(z / M r) P_{r}(z)^{2} r d z$

$$
=\int_{\mathbf{R}^{n}} \psi(z / M r) P_{r}(z)\left(|z|^{2}+r^{2}\right)^{(1-n) / 2} d z(1+O(\delta)) .
$$

Proof. Note that by looking at the special case of $(\dagger)$ in which $\varphi(X) \equiv$ 0 , we see that

$$
\frac{1}{\omega_{n}} \int_{\mathbb{R}^{n}} P_{r}(z)^{2} r d z=\int_{\mathbf{R}^{n}} P_{r}(z)\left(|z|^{2}+r^{2}\right)^{(1-n) / 2} d z
$$

Part (c) follows from this equation. For $M$ sufficiently large, multiply the integrands on the left hand side of (a) and (b) by $\psi(z / M r)$ and change the result by a factor at most $(1+\delta)$. The choice of $M$ depends only on the Lipschitz constant as in [10, Lemma 2.3 (a) and (c)]. Next, for $|z| \leq M r$,

$$
\left|\left\langle Q-X, N_{Q}\right\rangle\right| \leq|Q-X| \leq 2 M r .
$$

Therefore, using Lemma 4,

$$
\begin{aligned}
\int_{|z| \leq M r} \mid k_{X}(Q)^{2} & -P_{r}(z)^{2}||\left\langle Q-X, N_{Q}\right\rangle \mid d \sigma(Q) \\
& \leq \int_{|z| \leq M r} \frac{3 \varepsilon}{M} P_{r}(z)^{2} \cdot 2 M r \cdot(1+A) \frac{d z}{\alpha} \\
& \leq O(\varepsilon) \int P_{r}(z)^{2} r d z .
\end{aligned}
$$

Thus we can replace $k_{X}(Q)^{2}$ by $P_{r}(z)^{2}$. Now we calculate

$$
\begin{aligned}
\left\langle Q-X, N_{Q}\right\rangle d \sigma(Q)= & \left((\varphi(x), x)+r N_{a}\right) \cdot(-1, \nabla \varphi(x)) d x \\
= & \left(-\varphi(x)+x \cdot \nabla \varphi(x)+\frac{r}{\alpha}+\frac{r a \cdot \nabla \varphi(x)}{\alpha}\right) d x \\
= & {[x \cdot(\nabla \varphi(x)-a)-(\varphi(x)-a \cdot x)} \\
& \left.+\frac{r a}{\alpha} \cdot(\nabla \varphi(x)-a)+r \alpha\right] d x .
\end{aligned}
$$

Let $\left(m_{i j}\right)=$ id $-P+P / \alpha$. We will use the convention that repeated indexes are summed from 1 to $n$ in what follows. Thus $x_{i}=m_{i j} z_{j}$. For any $C^{1}$ function $f,\left(\partial / \partial z_{i}\right) f\left(x_{a}(z)\right)=f_{j}\left(x_{a}(z)\right) m_{j i}$. Therefore, $f_{k}\left(x_{a}(z)\right)=$ 
$\bar{m}_{i k}\left(\partial / \partial z_{i}\right) f\left(x_{a}(z)\right)$, where $m_{j i} \bar{m}_{i k}=\delta_{j k}$. Hence,

$$
\begin{aligned}
x \cdot(\nabla \varphi(x)-a) & =x_{i}\left((\varphi(x)-a x)_{i}\right) \\
& =m_{i j} z_{j}\left(\bar{m}_{k i}\left(\partial / \partial z_{k}\right)\right)\left(\varphi\left(x_{a}(z)\right)-a \cdot x_{a}(z)\right) \\
& =z_{k}\left(\partial / \partial z_{k}\right)\left(\varphi\left(x_{a}(z)\right)-a \cdot x_{a}(z)\right) .
\end{aligned}
$$

Therefore,

$$
\begin{aligned}
\int_{\mathbf{R}^{n}} & \psi(z / r M) P_{r}(z)^{2}[-(\varphi(x)-a \cdot x)+x \cdot(\nabla \varphi(x)-a) \\
& \left.+\frac{r a}{\alpha} \cdot(\nabla \varphi(x)-a)\right] d z / \alpha \\
= & \int_{\mathbf{R}^{n}} \psi(z / r M) P_{r}(z)^{2}\left[z_{k}\left(\partial / \partial z_{k}\right)\left(\varphi\left(x_{a}(z)\right)-a \cdot x_{a}(z)\right)\right. \\
& \left.-\left(\varphi\left(x_{a}(z)\right)-a \cdot x_{a}(z)\right)+\frac{r a_{i}}{\alpha} \bar{m}_{k i}\left(\partial / \partial z_{k}\right)\left(\varphi\left(x_{a}(z)\right)-a \cdot x_{a}(z)\right)\right] \frac{d z}{\alpha} \\
= & -\int_{\mathbf{R}^{n}}(n+1) \psi(z / r M) P_{r}(z)^{2}\left(\varphi\left(x_{a}(z)\right)-a \cdot x_{a}(z)\right) d z / \alpha \\
& -\int_{\mathbf{R}^{n}}\left[\left(\partial / \partial z_{k}\right) \psi(z / r M) P_{r}(z)^{2}\right]\left(z_{k}+\frac{r a_{i} \bar{m}_{k i}}{\alpha}\right) \\
& \times\left(\varphi\left(x_{a}(z)\right)-a \cdot x_{a}(z)\right) \frac{d z}{\alpha}
\end{aligned}
$$

This integral is majorized by a multiple of $\varepsilon \int_{R^{n}} P_{r}(z)^{2} r d z$. This proves part (a).

The proof of part (b) is similar, but simpler. We have $\left|Q_{a}(z)-Q(z)\right|^{2}=$ $\left|\varphi\left(x_{a}(z)\right)-a \cdot x_{a}(z)\right|^{2} \leq \varepsilon^{2} r^{2}$ for $|z| \leq M r$, and $\left|-r N_{a}-Q_{a}(z)\right|^{2}=$ $r^{2}+|z|^{2}$. Hence $\left|-r N_{a}-Q(z)\right|^{2}=\left(r^{2}+|z|^{2}\right)(1+O(\varepsilon))$ for $|z| \leq M r$. Thus $|X-Q|^{1-n}=\left(r^{2}+|z|^{2}\right)^{(1-n) / 2}(1+O(\varepsilon))$ and we can replace $\bar{k}_{X}(Q)$ by $P_{r}(z)$ as in part (a). This concludes the proof of Lemma 5 .

Now we can subtract (a) from (b) in Lemma 5 and using ( $f$ ) and Lemma 5(c) we find

$$
\begin{aligned}
\int_{\mathbf{R}^{n}} \psi(z / M r) P_{r}(z)\left(|z|^{2}+r^{2}\right)^{(1-n) / 2}\left(\sqrt{1+\left|\nabla \varphi\left(x_{a}(z)\right)\right|^{2}}-\alpha\right) \frac{d z}{\alpha} \\
\quad=O(\delta+\varepsilon) \int_{\mathbf{R}^{n}} P_{r}(z)\left(|z|^{2}+r^{2}\right)^{(1-n) / 2} d z=C_{n} r^{-n+1} O(\delta+\varepsilon) .
\end{aligned}
$$

Dividing by a suitable constant multiple of $r^{-n+1}$ we see that there is a dimensional constant $\alpha_{n}$ such that the function $g(z)=\alpha_{n}\left(1+|z|^{2}\right)^{-n} \psi(z / M)$ 
satisfies

$$
\begin{gathered}
\int_{\mathbf{R}^{n}} g(z) d z=1+O(\delta) \text { and } \\
\int g_{r}(z)\left(\sqrt{1+\left|\nabla \varphi\left(x_{a}(z)\right)\right|^{2}}-\sqrt{1+|a|^{2}}\right) d z=O(\delta+\varepsilon) \\
\text { with } g_{r}(z)=r^{-n} g(z / r) .
\end{gathered}
$$

Next, the same integration by parts used to prove Lemma $5(a)$ shows that

$$
\int g_{r}(z) a \cdot\left(\nabla \varphi\left(x_{a}(z)\right)-a\right) \frac{d z}{\alpha}=O(\delta+\varepsilon)
$$

Therefore,

where

$$
\int g_{r}(z) B\left(\nabla \varphi\left(x_{a}(z)\right), a\right) d z=O(\delta+\varepsilon)
$$

$$
B(v, a)=\sqrt{1+|v|^{2}}-\sqrt{1+|a|^{2}}-a \cdot(v-a) / \sqrt{1+|a|^{2}} .
$$

Define $\beta=\sqrt{1+|v|^{2}}$ and $\alpha=\sqrt{1+|a|^{2}}$, as before. Then

$$
\begin{aligned}
\frac{1}{2} \beta\left|\frac{(-1, v)}{\beta}-\frac{(-1, a)}{\alpha}\right|^{2} & =\frac{1}{2} \beta\left(1+1-2 \frac{(-1, v) \cdot(-1, a)}{\alpha \beta}\right) \\
& =\beta-(1+v \cdot a) / \alpha \\
& =\beta-\left(1+|a|^{2}+(v-a) \cdot a\right) / \alpha \\
& =B(v, a) .
\end{aligned}
$$

This identity can be stated in geometric form as

$$
B\left(\nabla \varphi\left(x_{a}(z)\right), a\right)=\frac{1}{2} \sqrt{1+\left|\nabla \varphi\left(x_{a}(z)\right)\right|^{2}}\left|N_{Q(z)}-N_{a}\right|^{2},
$$

but we will not use it in that form. Instead, we write

$$
\left|\frac{(-1, v)}{\beta}-\frac{(-1, a)}{\alpha}\right|^{2}=\left|\frac{1}{\beta}-\frac{1}{\alpha}\right|^{2}+\left|\frac{a}{\alpha}-\frac{v}{\beta}\right|^{2} \text {. }
$$

Thus

$$
\begin{aligned}
|v-a|^{2}=\beta^{2}\left|\frac{v}{\beta}-\frac{a}{\beta}\right|^{2} & \leq 2 \beta^{2}\left[\left|\frac{v}{\beta}-\frac{a}{\alpha}\right|^{2}+\left|\frac{a}{\alpha}-\frac{a}{\beta}\right|^{2}\right] \\
& \leq 2 \beta\left(2 B+2|a|^{2} B\right)=4 \alpha^{2} \beta B .
\end{aligned}
$$

We claim that either $|v-a| \leq \sqrt{\varepsilon+\delta}$ or $|v-a| \leq 12 \alpha^{3} B / \sqrt{\varepsilon+\delta}$. In fact, if $|v-a| \geq \sqrt{\varepsilon+\delta}$, then

$$
\frac{3 \alpha}{\sqrt{\varepsilon+\delta}}|v-a| \geq|v-a|+\alpha+1 \geq|v|-|a|+\alpha+1 \geq|v|+1 \geq \beta .
$$

Therefore,

$$
\beta|v-a| \leq \frac{3 \alpha}{\sqrt{\varepsilon+\delta}}|v-a|^{2} \leq \frac{3 \alpha}{\sqrt{\varepsilon+\delta}} \cdot\left(4 \alpha^{2} \beta B\right)
$$


which implies $|v-a| \leq 12 \alpha^{3} B / \sqrt{\varepsilon+\delta}$. Therefore, since $\int g(z) d z=O(1)$, we can split the integral that follows into two parts according to whether $\left|\nabla \varphi\left(x_{a}(z)\right)-a\right|$ is less than or greater than $\sqrt{\varepsilon+\delta}$ to obtain

$$
\begin{aligned}
\int g_{r}(z)\left|\nabla \varphi\left(x_{a}(z)\right)-a\right| d z \leq & \sqrt{\varepsilon+\delta} \int_{\mathbf{R}^{n}} g_{r}(z) d z \\
& +\frac{1}{\sqrt{\varepsilon+\delta}} \int_{\mathbf{R}^{n}} g_{r}(z) 12 \alpha^{3} B\left(\nabla \varphi\left(x_{a}(z)\right), a\right) d z \\
= & O(\sqrt{\varepsilon+\delta}) .
\end{aligned}
$$

Since the origin in this argument was arbitrary, we have proved $\nabla \varphi \in \mathrm{VMO}$.

6. Open questions. Harmonic measure is the measure on $\partial \Omega$ associated to a point $P \in \Omega$ such that

$$
u(P)=\int_{\partial \Omega} f(Q) d \omega^{P}(Q) \quad \text { for all } f \in C_{0}(\partial \Omega)
$$

where $u \in C_{0}(\bar{\Omega})$ is the solution to the Dirichlet problem $\Delta u=0$ in $\Omega$, $\left.u\right|_{\partial \Omega}=f$. In a Lipschitz domain $d \omega^{P}$ is mutually absolutely continuous with surface measure $d \sigma$, and $d \omega^{P} / d \sigma$ is the normal derivative of Green's function with pole at $P$ (defined almost everywhere).

A natural class of domains for potential theory is the class of nontangentially accessible (NTA) domains defined in [8]. If $\Omega$ is an unbounded NTA domain in $R^{n+1}$, then there is a positive harmonic function $u$ in $\Omega$ that vanishes on $\partial \Omega$, and $u$ is unique up to a constant multiple. This function should be viewed as Green's function at $\infty$. In the case $\Omega=\left\{\left(x_{0}, x\right): x_{0}>0\right\}$, $u\left(x_{0}, x\right)=c x_{0}$. The function $u$ determines a harmonic measure at $\infty, d \omega$, by the formula

$$
\int_{\partial \Omega} \varphi d \omega=\int_{\Omega} u \Delta \varphi \quad \text { for all } \varphi \in C_{0}^{\infty}\left(R^{n+1}\right) .
$$

The heart of Caffarelli's theorem (Theorem 3) is a Liouville-type corollary which says that if $\Omega$ is the region above the graph of a Lipschitz function and $u$ has normal derivative constant almost everywhere, then $\Omega$ is a half space and $u$ is linear. This can be rephrased as saying that if $d \omega=d \sigma$, then $\Omega$ is a half space.

On the other hand, Keldysh and Lavrent'ev costructed an example in the 1930's of an NTA domain in $\mathbf{R}^{2}$ which was not a half plane but for which $d \omega=d \sigma$. (See [5]. In the bounded case the goal is to show that $d \omega^{P}=d \sigma$ for some region other than a circle centered at $P$. The unbounded case is not treated explicitly, but follows from the same techniques.) Let us define 
a $K L$ domain to be an unbounded NTA domain for which $d \omega=d \sigma$. By taking a Cartesian product with a planar example, one obtains KL domains in any dimension that are not half spaces.

Caffarelli's theorem is analogous to Moser's theorem [16] that any Lipschitz graph that is a minimal hypersurface is necessarily a hyperplane. However, there are complete graphs (in $\mathbf{R}^{9}$ ) that are not hyperplanes, yet they are minimal hypersurfaces [2].

Problem 1. Does there exists a $K L$ domain $\Omega=\left\{\left(x_{0}, x\right): x_{0}>\varphi(x)\right\}$ with $\varphi$ not linear?

Problem 1'. Does there exist a $K L$ domain with $C^{\infty}$ boundary?

In $\mathbf{R}^{2}$ the answer is no because $C^{\infty}$ domains and regions above graphs have what is known as the Smirnov property (see [5]). Furthermore, Lavrent'ev proved that the chord-arc condition $\left({ }^{2}\right)$ suffices to rule out a $\mathrm{KL}$ domain.

However, in higher dimensions the situation is very different. Consider a function $u(\theta)$, defined in an open set $\omega \subset S^{n}$, the unit sphere in $\mathbf{R}^{n+1}$, satisfying $u>0$ and $\left(\Delta_{\theta}+n\right) u=0$ in $\omega, u=0$ on $\partial \omega$, and the normal derivative $\partial u / \partial \nu=$ constant on $\partial \omega$. (Here $\Delta_{\theta}$ denotes the spherical Laplacian: in polar coordinates $\left.\Delta=(\partial / \partial r)^{2}+(n / r) \partial / \partial r+\left(1 / r^{2}\right) \Delta_{\theta}.\right)$ It follows that $r u(\theta)$ is a positive harmonic function in the cone $\Omega=\{(r, \theta): \theta \in \omega\}$ which vanishes on $\partial \Omega$ and has constant normal derivative. One obvious solution to this problem is a degree one spherical harmonic $-r u(\theta)=a x_{0}$ in some coordinate system - and $\Omega$ is a half space. But there is another example, namely, $\omega$ can be a symmetric region about the equator formed by removing spherical caps about the north and south pole (see [1]). The solution $u$ can then be computed using rotational symmetry and ordinary differential equations. This example is the "enemy". It shows that the chord-arc condition or any comparable notion does not suffice to rule out the possibility of a domain other than the half space. Because of the "bad" cone, the best we can expect are "small constant" theorems (see Caffarelli [3], part II, and Alt and Caffarelli [1].)

Problem 2. Suppose that $\Omega$ is an unbounded $K L$ domain and for large $r$, $\sigma\left(B_{r} \cap \partial \Omega\right) \leq(1+\varepsilon) c_{n} r^{n}$, where $c_{n} r^{n}$ is the volume of the ball in $\mathbf{R}^{n}$ of radius $r, B_{r}$ is any ball in $\mathbf{R}^{n+1}$. Show that if $\varepsilon$ is sufficiently small depending on $n$, then $\Omega$ must be a half space.

There is a variational problem associated to constructing $\mathrm{KL}$ domains.

$\left({ }^{2}\right) \partial \Omega$ is said to satisfy the chord-arc condition if length $\left(\operatorname{arc}\left(z_{1}, z_{2}\right)\right) \leq C\left|z_{1}-z_{2}\right|$, for all $z_{1}, z_{2} \in \partial \Omega$, where $\operatorname{arc}\left(z_{1}, z_{2}\right)$ is the arc along $\partial \Omega$ from $z_{1}$ to $z_{2}$ and $\left|z_{1}-z_{2}\right|$ is the ordinary distance, or length of the chord from $z_{1}$ to $z_{2}$. 
One has to distinguish between critical points and absolute minima for this problem. The example of the cone built on the region $\omega$ symmetric about the equator is only a critical point and not a minimum for the functional, so there is still hope for a more general theorem for minimizers of this variational problem.

The procedure in [2] for constructing minimal graphs in $\mathbf{R}^{9}$ involves passing from minimal cones in $\mathbf{R}^{\mathbf{8}}$. In order to get started with the analogous question one needs to consider

Problem 3. For each $\lambda$, find all Lipschitz domains $\omega$ in $S^{n}$ such that there is a positive solution to $\Delta_{\theta} u+\lambda u=0$ in $\omega,\left.u\right|_{\partial \omega}=0$ and $\partial u /\left.\partial \nu\right|_{\partial \omega}=c$, a constant. ( $\lambda=n$ is the only case relevant to the problem in $\mathbf{R}^{n+1}$, and one needs to calculate which of these are minimal.)

Serrin [18] has shown that the only smooth domains $\Omega$ in $\mathbf{R}^{n}$ with solutions to $\Delta u+\lambda u=0$ and $u>0$ in $\Omega$ with $\left.u\right|_{\partial \Omega}=0, \partial u /\left.\partial \nu\right|_{\partial \Omega}=$ constant are balls. On the negative side we expect an analogue of the Keldysh-Lavrent'ev phenomenon:

Problem 4. Find a (nonsmooth) domain $\Omega$ in $\mathbf{R}^{2}$ and a solution to $u>0$ and $\Delta u+\lambda u=0$ in $\Omega,\left.u\right|_{\partial \Omega}=0, \partial u /\left.\partial \nu\right|_{\partial \Omega}=c$ almost everywhere on $\partial \Omega$.

(One could ask the similar question on the sphere.)

Finally; let us conclude with a nonlinear boundary value problem whose solution would imply Caffarelli's theorem.

Problem 5. Suppose that $\Omega$ is a Lipschitz domain, $\Delta u=0$ in $\Omega, c^{-1} \leq$ $N_{Q} \cdot \nabla u(Q) \leq c$ and $|\nabla u(Q)|^{2}$ belongs to $C^{\alpha}(\partial \Omega)$. Show that $\nabla u \in C^{\varepsilon}(\bar{\Omega})$ for some $\varepsilon>0$.

\section{REFERENCES}

[1] H. W. Alt and L. Caffarelli, Existence and regularity for a minimum problem with free boundary, J. Reine Angew. Math. 325 (1981), 105-144.

[2] E. Bombieri, E. de Giorgi, and E. Giusti, Minimal cones and the Bernstein problem, Invent. Math. 7 (1969), 243-268.

[3] L. Caffarelli, A Harnack inequality approach to the regularity of free boundaries, Parts I, II, III, Rev. Mat. Iberoamericana.

[4] B. E. J. Dahlberg, Estimates for harmonic measure, Arch. Rational Mech. Anal. 65 (1977), 275-288.

[5] P. Duren, The Theory of $H^{p}$ Spaces, Academic Press, New York 1970.

[6] G. B. Folland, Introduction to Partial Differential Equations, Math. Notes 17, Princeton Univ. Press, 1976.

[7] D. Gilbarg and N. S. Trudinger, Elliptic Partial Differential Equations of Second Order, Springer, Berlin 1978. 
[8] D. Jerison and C. E. Kenig, Boundary behavior of harmonic functions in nontangentially accessible domains, Adv. in Math. 46 (1982), 80-147.

[9] -, -, Boundary value problems on Lipschitz domains, in: W. Littmann (ed.), Studies in P.D.E., MAA Stud. Math. 23, 1982, 1-68.

[10] - , - The logarithm of the Poisson kernel of a $C^{1}$ domain has vanishing mean oscillation, Trans. Amer. Math. Soc. 273 (1982), 781-794.

[11] D. Kinderlehrer and L. Niren berg, Regularity in free boundary problems, Ann. Scuola Norm. Sup. Pisa (4) 4 (1977), 373-391.

[12] D. Kinderleh rer, L. Niren berg and J. S pruck, Regularity in elliptic free boundary problems, J. Analyse 34 (1978), 86-119.

[13] M. Lavrent'ev, Boundary problems in the theory of univalent functions, Mat. Sb. (N.S.) 1 (43) (1936), 815-844; English transl.: Amer. Math. Soc. Transl. (2) 32 (1963), 1-35.

[14] G. M. Lie be rm a n, Two-dimensional nonlinear boundary value problems for elliptic equations, Trans. Amer. Math. Soc. 300 (1987), 287-295.

[15] - Oblique derivative problems in Lipschitz domains, Boll. Un. Mat. Ital. B (7) 1 (1987), 1185-1210.

[16] J. Moser, On Harnack's theorem for differential equations, Comm. Pure Appl. Math. 14 (1961), 577-591.

[17] J. Pipher, Oblique derivative problems for the Laplacian in Lipschitz domains, Rev. Mat. Iberoamericana 3 (1987), 455-472.

[18] J. Serrin, A symmetry problem in potential theory, Arch. Rational Mech. Anal. 43 (1971), 304-318.

[19] E. M. S t ein, Singular Integrals and Differentiability Properties of Functions, Princeton Univ. Press, 1970.

[20] A. Z y g m u nd, Trigonometric Series, 2 vols., 2nd ed., Cambridge Univ. Press, 1959.

\section{DEPARTMENT OF MATHEMATICS}

M.I.T.

CAMBRIDGE, MASSACHUSETTS 02139, U.S.A. 